\title{
An Evaluation Model to Quantify the Qualitative Smart Growth in Medium Population City
}

\author{
Yanwan Huang, a \\ ${ }^{1}$ North China Electric Power University, Baoding071000, China; \\ aliusuanlvjia@163.com
}

Keywords: the analytic hierarchy process (AHP), the fuzzy evaluation method, smart growth, entropy method

\begin{abstract}
Our goal is to build a metric with indexes aimed to quantify the qualitative smart growth of a city. We formulate the problem of metric as a comprehensive evaluation model in which the success of smart growth is a specific value S. Primarily, based on the E's of sustainability and principles of smart growth, we select several indexes measuring the success of smart growth, and establish a three-tier structure of the evaluation indexes system. Secondly, the analytic hierarchy process (AHP) is applied to evaluate the weights of each tier. Besides, in order to overcome the subjectivity of AHP, this paper adapts the fuzzy evaluation method to improve model, and obtains the other set of weights. Then, the two sets of weights are combined with linear weighting methods to get the specific value S.
\end{abstract}

\section{Introduction}

Home is where the heart is, and community is where the home is. "Whether its causes are economic forces, consumer preferences, or misguided public policies, the dominant form of urban development over the postwar period can be characterized as urban sprawl."[1] So how to reduce the urban sprawl with urban development is the problem that smart growth wants to solve.

The ten principles for smart growth that have gained widespread recognition are: [1]

- Create a range of housing opportunities and choices;

- Create walkable neighborhoods;

- Encourage community and stakeholder collaboration;

- $\quad$ Foster distinctive, attractive places with a strong sense of place;

- Make development decisions predictable, fair, and cost-effective;

- Mix land uses;

- $\quad$ Preserve open space, farmland, natural beauty, and critical environmental areas;

- Provide a variety of transportation choices;

- $\quad$ Strengthen and direct development towards existing communities;

- Take advantage of compact building design.

An optimal evaluation model is required for the communities to determine whether their growth plan conforms to smart growth.

\section{Quantify the success of smart growth}

\subsection{Data Collection and Data Standardization}

We determine the hierarchical structure diagram of our evaluation mode, and they are shown in Fig.1. 


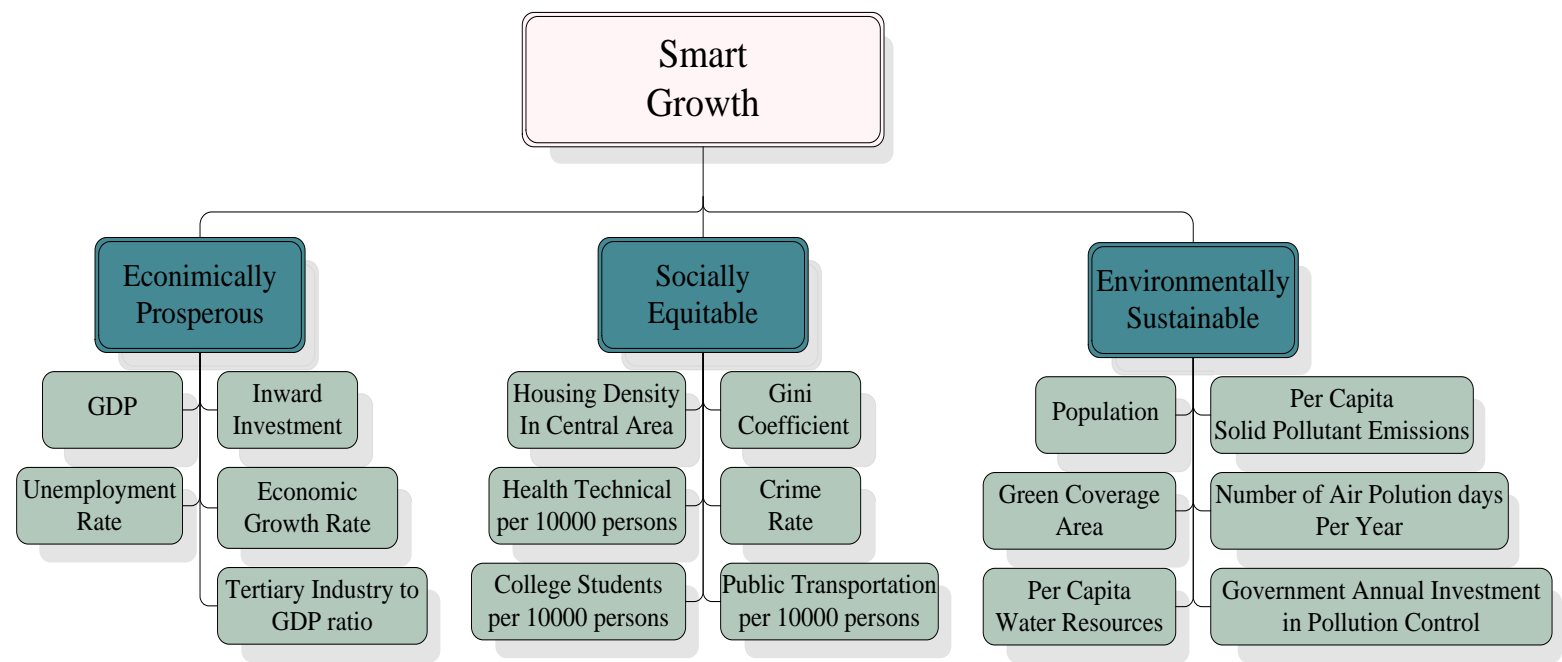

Fig.1: The hierarchical structure diagram of the indexes of our evaluation model.

Consequently, we choose five typical cities as samples: Canberra, Karamay, Liverpool, Parreira, and Markham. According to the influence of the indexes on the success rate of smart growth, we adopt two standardized formulas to make the data standardized. For positive indexes we use formula (1) to standardize them:

$$
b_{S i}=\frac{b_{i} n}{\sum_{j=0}^{n} b_{i j}}
$$

For passive indexes we use formula (2) to standardize them:

$$
b_{S i}=\frac{\sum_{j=0}^{n} b_{i j}}{b_{i} n}
$$

\subsection{Weight Analysis}

$\diamond$ Determine the judging matrix: we determine the judging matrix by one-nine method ${ }^{[3]}$.

$\diamond$ Determine the weight vector: the greatest eigenvalue of matrix A,B is $\lambda_{m}$, and the corresponding eigenvector is $\bar{W}=\left(\begin{array}{llll}\bar{w}_{1} & \bar{w}_{2} & \cdots & \bar{w}_{n}\end{array}\right)^{T}$. Then we calculate the normalized eigenvector by the following formula:

$$
w_{i}=\frac{\bar{w}_{i}}{\sum_{i=0}^{n} \bar{w}_{i}}, i=1,2, \cdots, n
$$

So the formula to evaluate the success of smart growth of a city is:

$$
\begin{aligned}
S_{1} & =A W \times \mathrm{a} \\
& =a w_{1} \times a_{1}+a w_{2} \times a_{2}+a w_{3} \times a_{3} \\
& =a w_{1} \times B W_{1} \times \mathrm{b}^{1}+a w_{2} \times B W_{2} \times \mathrm{b}^{2}+a w_{3} \times B W_{3} \times \mathrm{b}^{3}
\end{aligned}
$$

Where:

$$
\mathrm{b}^{1}=\left[\begin{array}{llll}
b_{1} & b_{2} & \cdots & b_{5}
\end{array}\right]^{T}, \mathrm{~b}^{2}=\left[\begin{array}{llll}
b_{6} & b_{7} & \cdots & b_{11}
\end{array}\right]^{T}, \mathrm{~b}^{3}=\left[\begin{array}{llll}
b_{12} & b_{13} & \cdots & b_{17}
\end{array}\right]^{T} .
$$

\subsection{Numerical Calculation}

Based on the conclusion in section 2.2, we reach the following results:

- According to data colleted, we determin the judging matrix, and get the weight vector of it:

$$
\begin{gathered}
A W=\left[\begin{array}{lllll}
0.1396 & 0.5279 & 0.3325
\end{array}\right] \\
B W_{1}=\left[\begin{array}{llllll}
0.1093 & 0.1093 & 0.2063 & 0.2063 & 0.3689
\end{array}\right] \\
B W_{2}=\left[\begin{array}{llllll}
0.250 & 0.083 & 0.083 & 0.250 & 0.083 & 0.250
\end{array}\right]
\end{gathered}
$$




$$
B W_{3}=\left[\begin{array}{llllll}
0.1148 & 0.1148 & 0.3242 & 0.3242 & 0.0610 & 0.0610
\end{array}\right]
$$

\subsection{Fuzzy Analysis}

To reduce the subjectivity of the Analytic hierarchy process (AHP), we improve our model by fuzzy theory. Then we get another set of weights.

$\diamond$ Constructing data matrix: We form the $m \times n$ matrix $B_{m \times n}$ of the data. Where the total of indexes is $m$, the total of cities is $n$. And we define the standardized data matrix as $B_{S}$.

$\diamond$ Obtain the fuzzy matrix: Based on the trichotomy of fuzzy theory a membership function was formed, in which $V_{m \times n}$ is the fuzzy matrix. We divide the interval from the minimum to the maximum of each line in $B_{S}$ into four part averagely. Then we have interval1 to interval 4 .

$$
\left\{\begin{array}{l}
V_{i j}=0, B_{S} \in \text { interval1 } \\
V_{i j}=1, B_{S} \in \text { interval } 2 \\
V_{i j}=2, B_{S} \in \text { interval3 } \\
V_{i j}=3, B_{S} \in \text { interval } 4
\end{array}\right.
$$

$\diamond$ Calculate the entropy value: According to previous steps, we get the fuzzy matrix $V$ of the sub-criterion layer. Analogously, we have the divided fuzzy matrix $D_{k j}$ of the three criterion later, then we have the complete fuzzy matrix $D_{3 \times n}$, and the transpose of this matrix is $X$. On the basis of entropy method, we calculate the proportion of the $j^{\text {th }}$ city's $k^{\text {th }}$ index by:

$$
p_{j k}=\frac{X_{j k}}{\sum_{j=1}^{n} X_{j k}}
$$

Then we have the $k^{\text {th }}$ index's entropy weight:

$$
\omega_{j}=\frac{\left(1+\frac{1}{\ln n} \sum_{k=1}^{3} p_{j k} \cdot \ln p_{j k}\right)}{\sum_{j=1}^{m}\left(1+\frac{1}{\ln n} \sum_{k=1}^{3} p_{j k} \cdot \ln p_{j k}\right)}
$$

Choosing two cities from $n$ cities, and we also then have the fuzzy matrix $X_{C}$ in which there are 3 columns and 2 lines. Finally, we got the success of smart growth:

\subsection{Combination Model}

$$
S_{2}=\omega \times \frac{X_{C}}{\frac{\sum_{j=1}^{n} X}{n}}
$$

Overall, the AHP method has the disadvantages that it's unconvincing with less quantitative data used and relatively more qualitative analysis. Besides, it's a simple and practical method to weight indexes. Reversely the Fuzzy Evaluation method is good at quantitative objects-evaluating. To integrate it, we get:

\subsection{Final results}

$$
S=0.5 S_{1}+0.5 S_{2}
$$

Applying model above to the data collected, the calculation results are showed as follows. 
Table 6: The final result

\begin{tabular}{cccccc}
\hline & $\begin{array}{c}\text { Liverpoo } \\
\mathbf{l}\end{array}$ & Karamay & Standard & AW & ( \\
\hline $\begin{array}{c}\text { Economic } \\
\text { Prosperity } \\
\begin{array}{c}\text { Socially } \\
\text { Equitable }\end{array}\end{array}$ & 8 & 9 & 8 & 0.1396 & 0.2630 \\
$\begin{array}{c}\text { Environmentally } \\
\text { sustainable }\end{array}$ & 8 & 5 & 9.4 & 0.3325 & 0.3366 \\
$\mathbf{S}_{\mathbf{1}}$ & 1.0843 & 0.8939 & 1.0000 & - & - \\
$\mathbf{S}_{\mathbf{2}}$ & 1.1383 & 0.7890 & 1.0000 & - & - \\
\hline $\mathbf{S}$ & 1.1113 & 0.8415 & 1.0000 & - & - \\
\hline
\end{tabular}

\section{Literature References}

The three E's of sustainability embody economic prosperity, social equity, and environmental sustainability and smart growth focus on building cities that embrace the three E's.

As is shown in the literature [2], the indexes to evaluate economy based on smart growth consist of GDP, jobless rate, economic growth rate, inward investment and tertiary industry output ratio of GDP.

There are some methods to establish the evaluation model [3].one is the Analytic Hierarchy Process (AHP), which was established by Thomas L. Saaty in the 1970s. The AHP is an effective method that transforms the half-qualitative and half-quantitative questions into quantitative questions. The Fuzzy Synthetic Evaluation is a method based on fuzzy mathematic, and applied to evaluate the object quantifiably.

\section{Summary}

Generally, Liverpool is successful in Smart Growth compared with our standard, Karamay is unsuccessful in Smart Growth compared with our standard. For Smart Growth, social equity is the most significant factor. Liverpool, a coastal city in the UK, has a maritime climate. Liverpool lacks mineral resources but its foreign trade is very developed. Its ratio of tertiary industry to economic is very large. However, as a city of a developed country, its industrial structure has been unchanged for such a long time that the jobs having existed cannot meet the growth of employment demand, resulting in that, the unemployment rate is very high. Superior geographical conditions bring both opportunities and challenges.

As for Karamay, it is an inland city with abundant oil resources. A typical continental climate makes it short of water. Most of the landforms are Gobi. At present, Karamay is still a single-industrial city dominated by oil industry. However, China's policy which named Silk Road Economic Zone brings greater market and economic opportunities to Karamay.

\section{References}

[1]. Gerrit Knaap, Emily Talen. New Urbanism and Smart Growth: a Few Words from the Academy. International Regional Science Review. Vol. 28 (2005) No. 2, p. 107-118.

[2]. Todd Litman. Understanding Smart Growth Savings What We Know about Public Infrastructure and Service Cost Savings, And How They are Misrepresented By Critics. Victoria Transport Policy Institute. (2004)

[3]. Zhonggeng Han, Yiqing Lu, Sujing Zhou. Mathematical Modeling and Practical Course. Higher Education Press, 2012, p.169 -170. 